\title{
Physical mechanism of spontaneous fast reconnection evolution
}

\author{
M. Ugai \\ Department of Computer Science, Faculty of Engineering, Ehime University, Matsuyama 790-8577, Japan
}

(Received May 26, 2000; Revised October 26, 2000; Accepted December 5, 2000)

\begin{abstract}
Large dissipative events, such as solar flares and geomagnetic substorms, result from sudden onset of magnetic reconnection, so that it is a long-standing problem to find the physical mechanism that makes magnetic reconnection explosive. As recognized by Petschek, standing slow shocks enable the effective magnetic energy conversion in space plasmas of extremely large magnetic Reynolds number. Hence, a basic question is how the fast reconnection mechanism involving slow shocks can be realized as an eventual solution? We have proposed the spontaneous fast reconnection model, which describes a new type of nonlinear instability that grows by the positive feedback between plasma microphysics (current-driven anomalous resistivity) and macrophysics (global reconnection flow). It is demonstrated that the fast reconnection mechanism explosively grows by the positive feedback in a variety of physical situations; for the larger threshold of anomalous resistivity, the fast reconnection evolves more drastically. Also, distinct plasma processes, such as large-scale plasmoid and magnetic loop dynamics, result directly from the fast reconnection evolution. Even in general asymmetric situations, the spontaneous fast reconnection model effectively works, giving rise to drastic magnetic flux transfer.
\end{abstract}

\section{Introduction}

It is known that solar flares and geomagnetic substorms result from sudden transition from an equilibrium of critically stored magnetic energy to another one of lower magnetic energy (Kulsrud, 1998). Hence, a question is: How can the magnetic energy be explosively converted into plasma energy in space plasmas of extremely large magnetic Reynolds number? In this respect, satellite observations have clearly indicated that magnetic reconnection plays an essential role in flare phenomena (e.g., Tsuneta, 1996). In space plasmas, magnetic energy should be released most effectively by the motor effect, $\mathbf{u} \cdot(\mathbf{J} \times \mathbf{B})>0$, and such a large-scale motor mechanism can be provided by standing slow shocks (Petschek, 1964). Therefore, the fast reconnection mechanism responsible for flares should involve slow shocks standing in a large-scale region.

In usual circumstances, the effective resistivity is extremely small, so that strong current systems in the form of current sheets are likely to be formed in space plasmas, leading to an equilibrium of critically stored magnetic energy. Then, a basic question is: Coupled to nonideal effects, how can the fast reconnection mechanism suddenly evolve and be realized as an eventual solution? In this respect, the conventional 'externally driven fast reconnection model' predicts that external boundary conditions should control the fast reconnection evolution and hence determine its fundamental configuration; accordingly, the effective resistivity has no fundamental effect on the fast reconnection evolution if there is any finite resistivity (Petschek, 1964; Vasyliunas, 1975; Sato and Hayashi, 1979; Priest and Forbes, 1986).

Copy right (c) The Society of Geomagnetism and Earth, Planetary and Space Sciences (SGEPSS); The Seismological Society of Japan; The Volcanological Society of Japan; The Geodetic Society of Japan; The Japanese Society for Planetary Sciences.
On the other hand, we have proposed the spontaneous fast reconnection model, which describes a new type of nonlinear instability in a long current sheet system (Ugai, 1984, 1986, 1999a). The basic idea lies in the following positive feedback: That is, the self-consistent coupling between (microscopic) current-driven anomalous resistivity and (macroscopic) reconnection flow gives rise to simultaneous growth of localized resistivity and fast reconnection flow by each enhancing the other. An important point is that the anomalous resistivity is localized at the $\mathrm{X}$ neutral point, which allows the fast reconnection to evolve effectively (Ugai and Tsuda, 1977; Scholer, 1989a). In fact, for various current-driven anomalous resistivity models many MHD simulations have demonstrated that the fast reconnection evolution can be realized by the positive feedback (Scholer, 1989b; Yokoyama and Shibata, 1994; Schumacher and Kliem, 1996; Magara and Shibata, 1999; Shibata, 1999).

An effective resistivity at an X neutral point, required for magnetic reconnection to take place, results from plasma microphysics, such as Coulomb collisions, nonadiabatic particles (inertial effects), and (current-driven) microinstabilities. Of course, MHD studies cannot treat the microphysics and are hence obliged to assume an effective resistivity in terms of macroscopic quantities. But, in order to understand the global (macroscopic) features of magnetic reconnection and the associated flare phenomena, MHD treatments should be very important. In fact, high-frequency microscopic plasma processes may often allow a collisionless plasma to behave as a fluid. Hence, in what follows, the spontaneous fast reconnection evolution and the associated distinct plasma processes will be demonstrated by high-resolution MHD simulations. 


\section{Spontaneous Fast Reconnection Model}

First of all, let us present the basic concept of the spontaneous fast reconnection model (Ugai, 1999a). In order to see the key points, the two-dimensional simulation model is simplified as follows. As an initial configuration in static equilibrium, a one-dimensional current sheet system with symmetric antiparallel ( $x$ directional) magnetic fields (of magnitude $\left.B_{x 0}=1\right)$ is assumed, where the pressure-balance condition, $P(y)+B_{x}^{2}(y)=1+\beta_{0}$, is satisfied (plasma pressure $P$ is normalized by $\left.B_{x 0}^{2} /\left(2 \mu_{0}\right)\right)$ and the normalized temperature $T(=P / \rho)$ is constant everywhere (plasma density $\rho$ is normalized by $\rho_{0}$ measured at the center of the current sheet (at $y=0)$ ); also, low- $\beta$ plasma $\left(\beta_{0}=0.15\right)$ is assumed in the magnetic field region. Here, distances are normalized by the half-width of the current sheet $d_{0}$, B by $B_{x 0}$, and time $t$ by $d_{0} / V_{A x 0}\left(V_{A x 0}=B_{x 0} / \sqrt{\mu_{0} \rho_{0}}\right)$. Also, the initial antiparallel magnetic field is assumed to be of a finite width in the upstream computational region, so that the current at the upper boundary of the magnetic field flows in the direction opposite to the one in the central current sheet $(|y|<1)$; hence, the total current integrated over the whole computational region is zero initially. Since distinct phenomena build up from near the X point and propagate outwards with time, the total current remains to be nearly zero until the magnetic field changes significnatly at the outermost boundaries because of the Ampere's law.

In order to disturb the initial configuration, a localized resistivity is artificially imposed at the origin only in the initial (normalized) time range $0<t<4$. After the initial disturbance is removed, a current-driven anomalous resistivity model is assumed for $t>4$ typically as

$$
\begin{aligned}
\eta(\mathbf{r}, t) & =k_{R}\left[V_{d}(\mathbf{r}, t)-V_{C}\right] & & \text { for } V_{d}>V_{C}, \\
& =0 & & \text { for } V_{d}<V_{C},
\end{aligned}
$$

where $V_{d}=|\mathbf{J}(\mathbf{r}, t) / \rho(\mathbf{r}, t)|$ is the relative electron-ion drift velocity, and $V_{C}$ is its threshold for a microinstability (here, $k_{R}=0.003$ is taken). Also, the threshold $V_{C}$ is assumed to increase with the temperature $T$ as

$$
V_{C}(\mathbf{r}, t)=V_{C 0}\left[T(\mathbf{r}, t) / T_{0}\right]^{\alpha},
$$

where $T_{0}=1+\beta_{0}$ is the initial uniform temperature, and $0 \leq \alpha \leq 1$ may be taken.

We find that if $V_{C 0}$ is sufficiently large, the fast reconnection mechanism can explosively evolve. Figure 1 shows the temporal behaviors of quantities for $\alpha=0.5$ and $V_{C 0}=4$, where $V_{C}$ increases with the thermal velocity. Initiated by the finite resistivity $\eta$ as an initial disturbance, the plasma flow grows so as to enhance the drift velocity $V_{d}$ at the origin, so that the threshold $V_{C}$ is eventually exceeded and the anomalous resistivity (1) suddenly occurs at time $t \sim 10$. Accordingly, magnetic reconnection (or the electric field $E(\mathbf{r}=0)$ ) grows slowly for a while and then grows explosively (more than exponentially) in the time range $20<t<27$. Obviously, this explosive reconnection growth results from the drastic positive feedback between the reconnection flow and the anomalous resistivity. The resulting peak reconnection rate is sustained during the nonlinear saturation phase $(t>$ 30).

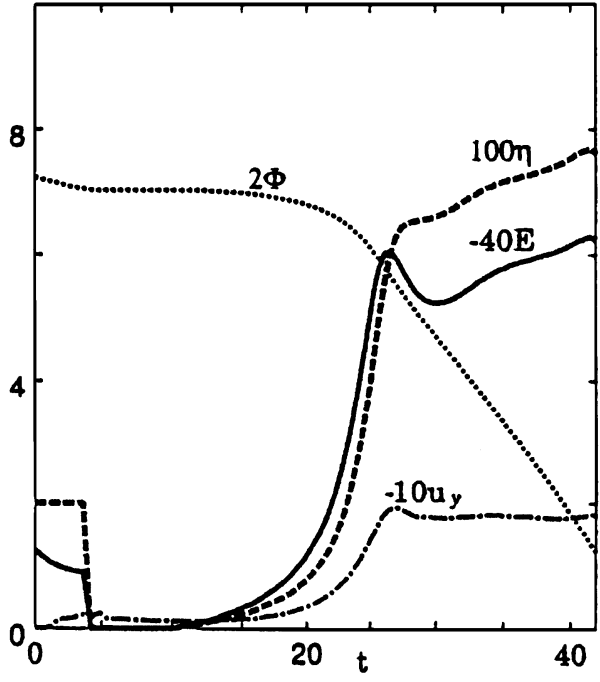

Fig. 1. Temporal variations of the electric field $E(\mathbf{r}=0)$, the resistivity $\eta(\mathbf{r}=0)$, the inflow velocity $u_{y}(x=0, y=0.9)$, and the magnetic flux $\Phi(t)$ for the case of $V_{C 0}=4$ and $\alpha=0.5$.

The positive feedback mechanism is found to occur in the following manner. Note that plasma flow is initially zero everywhere and grows through the reconnection process. Then, localized anomalous resistivity causes magnetic reconnection, and the resulting $\mathbf{J} \times \mathbf{B}$ force tends to eject the plasma from near the $\mathrm{X}$ neutral point because of the accelerated outflow velocity $u_{x}$; accordingly, the ambient magnetized plasma is sucked into the $\mathrm{X}$ point, accelerating the inflow velocity $u_{y}$ (Fig. 1) so as to enhance the current density at the $\mathrm{X}$ point because of the frozen-in condition. Therefore, the reconnection flow grows so as to enhance the drift velocity $V_{d}$ and thus the anomalous resistivity (1) locally at the $\mathrm{X}$ point, leading to further enhancement of magnetic reconnection. In this way, the reconnection flow and the anomalous resistivity can simultaneously grow by each enhancing the other, so that this physical process is called the 'positive feedback'. In fact, Fig. 1 indicates that once the flow velocities have grown sufficiently large and the nonlinear effects become dominant for $t>20$, the positive feedback occurs explosively. However, the reconnection rate cannot grow indefinitely, since the increasing magnetic diffusion due to the enhanced resistivity tends to reduce the current density (anomalous resistivity) at the $\mathrm{X}$ point. Hence, when the anomalous resistivity has grown so large that the magnetic diffusion becomes balanced with the magnetic field convection by the plasma inflow $u_{y}$, the fast reconnection evolution becomes nonlinearly saturated for $t>30$.

We also find that the fast reconnection evolution is insensitive to the resistivity parameter $\alpha$ in (2), despite that the temperature should distinctly increase at the X neutral point. For instance, Fig. 2 shows the temporal variations of the reconnection rate $E(\mathbf{r}=0)$ and indicates that the resulting (peak) reconnection rate during the nonlinear saturation phase (for $t>30$ ) is almost the same for both $\alpha=0$ and 1. This demonstrates that even if the threshold of anomalous resistivity increases significantly with the temperature, the reconnection flow can grow so powerfully that the positive 


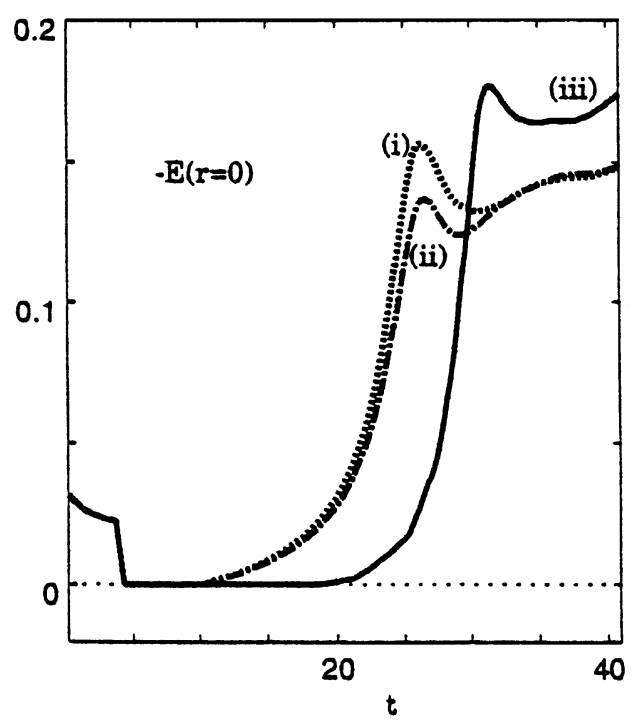

Fig. 2. Temporal behaviors of the electric field $E(\mathbf{r}=0, t)$ for the cases (i) $\alpha=0\left(V_{C 0}=4\right)$, (ii) $\alpha=1\left(V_{C 0}=4\right)$ and (iii) $V_{C 0}=12(\alpha=0.5)$.

feedback mechanism works effectively to enhance and sustain the anomalous resistivity locally at the X point. Also, the fast reconnection evolution is more drastic for the larger $V_{C 0}$ (Fig. 2), since for the larger $V_{C 0}$ the resulting anomalous resistivity is more effectively localized at the $\mathrm{X}$ point. We may hence understand that when an anomalous resistivity is far from occurring in usual circumstances, the fast reconnection mechanism can evolve explosively.

Figure 3 shows the resulting magnetic field configuration for the case of $V_{C 0}=12(\alpha=0.5)$ during the nonlinear saturation phase of the fast reconnection evolution (Fig. 2), where a pair of thin current layers indicate slow (switch-off) shocks (here, the computational region is restricted to the first quadrant only because of the conventional symmetry boundary conditions on the $x$ and $y$ axes). Apparently, the spontaneous fast reconnection mechanism during the nonlinear saturation phase may be characterized by the localized resistivity (diffusion region), the extending slow shocks, and the propagating large-scale plasmoid. As seen from Figs. 1 and 2, the attained peak reconnection rate $(|E|)$ as well as the peak anomalous resistivity is sustained and becomes almost constant with time; hence, if the reconnection process near the diffusion region is considered, it may be regarded as a quasi-steady process during the nonlinear saturation phase. Of course, if the overall system is considered, the system dynamics like the plasmoid propagation is strongly time-dependent, leading to drastic collapse of the large-scale magnetic field configuration; in fact, Fig. 1 shows that the total magnetic flux $\Phi(t)$ residing in the upper half region (defined as the line integral of $B_{x}(x=0, y, t)$ along the $y$ axis) drastically decreases during the nonlinear saturation phase.

We also find that, if a uniform resistivity is assumed for $t>4$, the reconnection rate grows at a small rate and the explosive positive feedback cannot appear. After the reconnection process is nonlinearly saturated, the peak reconnection rate is not sustained but simply decays with time. The central diffusion region is elongated with time, leading to the

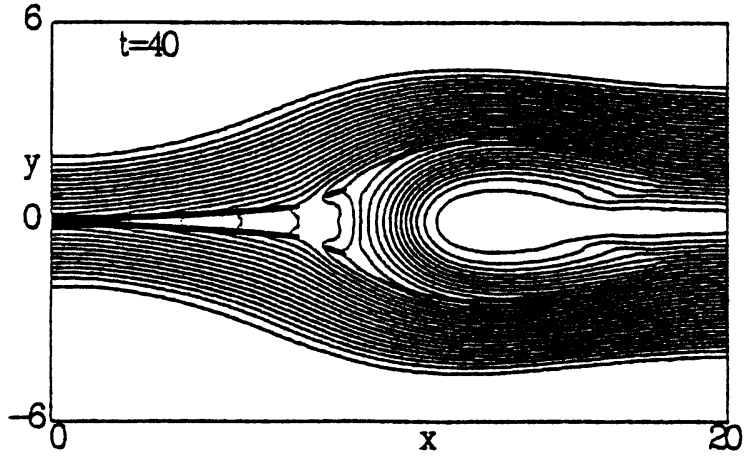

Fig. 3. Magnetic field configuration during the nonlinear saturation phase for the case of $V_{C 0}=12(\alpha=0.5)$, where the contour lines of current density (for $|J|>2.8$ ) are also indicated.

Sweet-Parker mechanism (without any standing slow shock). We hence conclude that the self-consistent interaction with the (current-driven) anomalous resistivity should be essential for the explosive evolution of fast reconnection mechanism.

\section{Consequences of the Spontaneous Fast Recon- nection Evolution}

A variety of distinct plasma processes result directly from the spontanous fast reconnection evolution. Here, the following three topics are demonstrated, which may be fundamental in understanding very complicated solar and terrestrial phenomena.

\subsection{Large-scale plasmoid dynamics}

It is well known from satellite observations that a largescale plasmoid propagates in the distant geomagnetic tail. As already stated, formation of a large-scale plasmoid is the direct outcome of the spontaneous fast reconnection evolution during the nonlinear saturation phase. Here, some basic plasmoid features, associated with this theoretical model, may be summarized as follows.

One is concerned with the magnetic field topology in the plasmoid. The conventional classical cartoon models have predicted that the field lines are essentially closed in the plasmoid. On the other hand, we have demonstrated that the field lines in the plasmoid are not closed but are open to outer free space: That is, they must not be necessarily closed in the plasmoid (Fig. 3), if a large-scale plasmoid develops in an initially one-dimensional current sheet (Ugai, 1995). Of course, if there is a normal field component in the initial current sheet, the associated reconnected field lines may be closed in the plasmoid. We also find that slow shocks stand along the plasmoid boundary. In the backward half of the plasmoid boundary, the motor effect works effectively, releasing the magnetic energy stored in the ambient region. On the other hand, the generator effect works in the forward half of the plasmoid, so that the region where magnetic field magnitude is notably enhanced propagates with the plasmoid.

The second point is concerned with the characteristics of plasmoid propagation. In the classical cartoon model, it was considered that a plasmoid propagated simply as a fireball, whereas we have seen that the plasmoid propagation apparently has wave characteristics. In order to examine this, computer simulations have been done by placing fluid ele- 


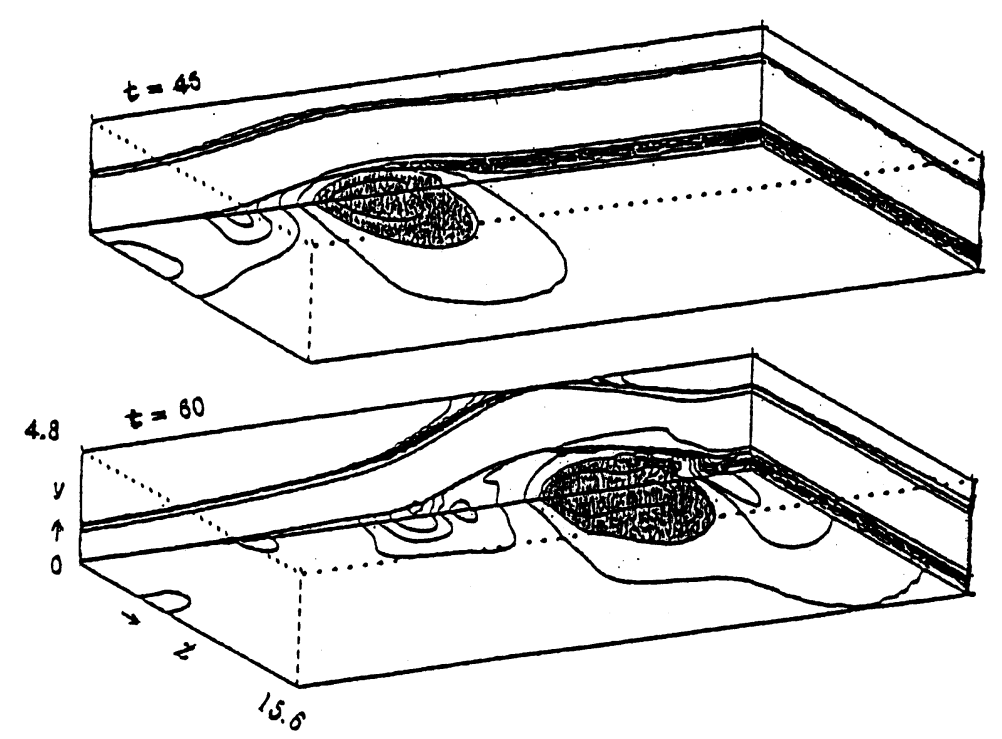

Fig. 4. Plasma pressure distributions of three-dimensional plasmoid with the anomalous resistivity model (1).

ments ahead of the plasmoid both in the central current sheet and in the ambient magnetic field region. Here, each fluid element is forced to move with the fluid velocity measured at the spatial point of the fluid element, which is obtained from the MHD simulation. Of course, the fluid elements stay almost stationary at the initial locations before the plasmoid arrives. We then find that, as the plasmoid is passing through, the fluid elements enter deeply into the plasmoid. This fact apparently indicates that the plasmoid propagates as a large-amplitude wave.

Finally, the two-dimensional plasmoid dynamics (Ugai, 1995 ) can readily be extended to the $z$ direction (three dimensions) (Ugai and Wang, 1998). For instance, Fig. 4 shows the three-dimensional simulation results that indicate the plasma pressure distributions at different times. In this figure, the three-dimensional plasmoid is identified with the region of notably enhanced plasma pressure (shaded in the figure). We see that the plasmoid remains confined in the $z$ direction because of the global vortex flow, so that the plasmoid propagates in the similar manner as in the two-dimensional case. We also find that the significant shear flow at the plasmoid side boundary gives rise to a notable sheared magnetic field component $B_{z}$ there.

\subsection{Magnetic loop dynamics}

If the plasmoid propagation is braked by some obstruction, the reconnected field lines should be piled up, so that a large-scale magnetic loop may be formed. With this in mind, we initially assume a long current sheet where a wall boundary is placed on the left side boundary. Initiated by a disturbance at $x=x_{d}$, the spontaneous fast reconnection evolution gives rise to a pair of plasmoids propagating in the positive and negative $x$ directions. The plasmoid propagating in the negative $x$ direction collides with the left side wall boundary, eventually giving rise to a large-scale magnetic loop. Then, distinct plasma processes result from the selfconsistent interaction between the strong fast reconnection jet and the magnetic loop (Ugai, 1996, 1999b).

If the reconnection site $\left(x \sim x_{d}\right)$ is sufficiently far from the left wall boundary, the reconnection jet towards the loop becomes supersonic (superfast), so that the collision with the magnetic loop gives rise to a definite fast shock ahead of the loop top. For the case $x_{d}=20$ Fig. 5 shows the temporal behaviors of the fast shock Mach number $M$ and the shock location $X_{f}$ and indicates that the shock strength oscillates as the magnetic loop swells further and the fast shock moves in the positive $x$ direction. The oscillation may result from the reflection of waves from the left boundary. Finally, the fast shock decays in a few tens of normalized time, when the fast shock location becomes close to the diffusion region.

The detailed structure of the large-scale magnetic loop at time $t=81$ is shown in Fig. 6. As can be seen, the superfast reconnection jet in the narrow wedge-like channel between a pair of slow shocks drastically collides with the magnetic loop and gives rise to a magnetic hollow at the loop top of the shape of "W". The fast shock stands only to a very limited extent in front of the loop top, and the complicated plasma flow behind the shock is finally directed down to the footpoints along the loop boundary. We in fact see that there are three spots of remarkably enhanced plasma pressure, at the loop top as well as at the pair of footpoints. We also find that the "quasi-steady" fast reconnection mechanism on the nonlinear saturation phase is little influenced by the magnetic loop dynamics. This may be because the reconnection jet is superfast, so that disturbances at the loop top cannot propagate back to and influence the reconnection (diffusion) region.

The two-dimensional loop dynamics can readily be extended to three dimensions in the $z$ direction. Let the $(y, z)$ plane be a wall plane as in the two-dimensional case, and let an initial three-dimensional disturbance be imposed at $x=x_{d}$ in the current sheet to a limited extent in the $z$ direction. Recently, we have performed the three-dimensional simulations for the case of $x_{d}=10$, and Fig. 7 illustrates the resulting plasma flow configurations near the magnetic loop. As can be observed from this figure, the fast reconnection jet towards the loop top remains significantly confined in the 


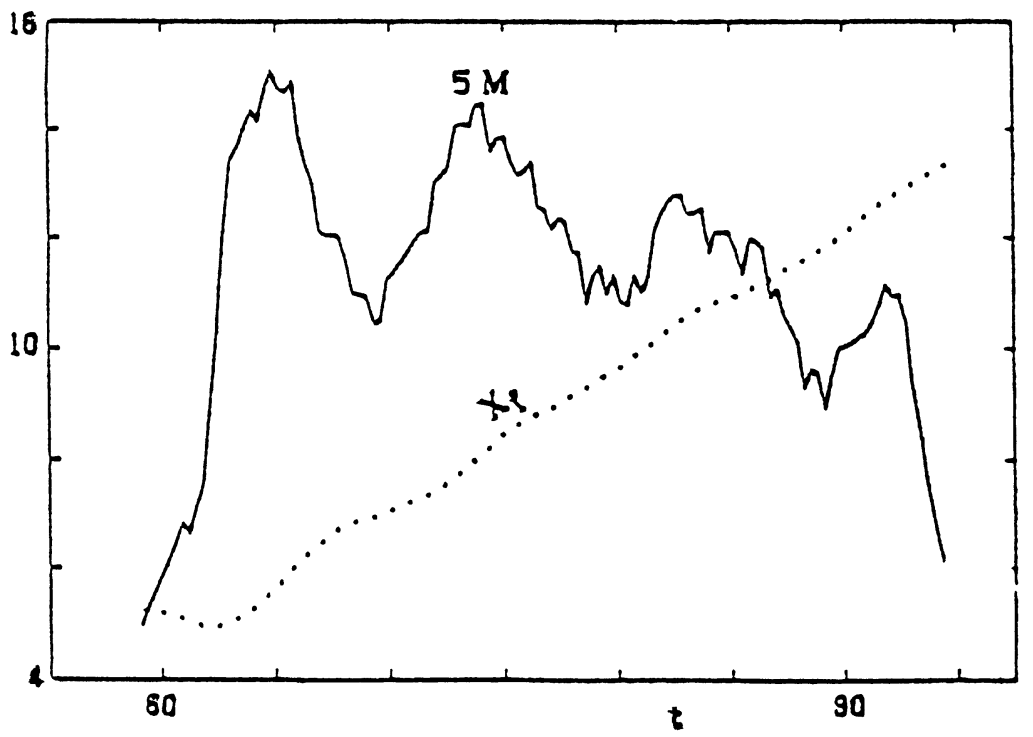

Fig. 5. Temporal behaviors of the fast shock Mach number $M$ and the shock location $X_{f}$ for the case of $x_{d}=20$.
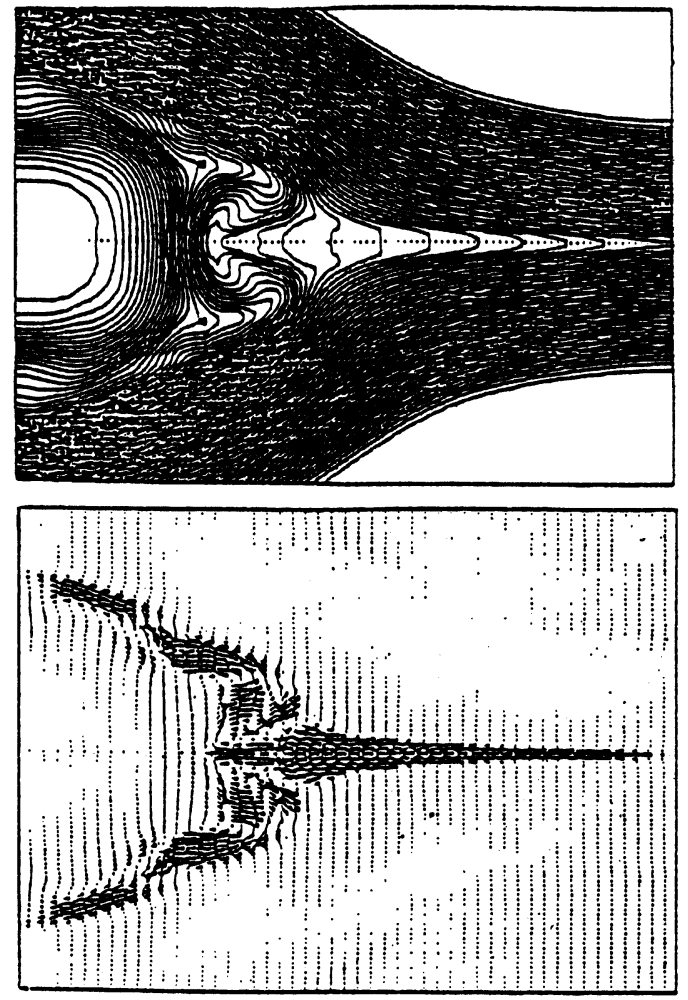

Fig. 6. Detailed magnetic field and plasma flow configurations at $t=81$ near the magnetic loop for the case of $x_{d}=20$.

$z$ direction because of the resulting vortex flow. Hence, we again find that the fast reconnection jet becomes superfast, giving rise to a fast shock ahead of the magnetic loop top, as in the two-dimensional case.

\subsection{Asymmetric fast reconnection evolution}

There are many important situations where antiparallel fields of different magnitudes, which are connected to different magnetic dipole sources, are reconnected. For instance,

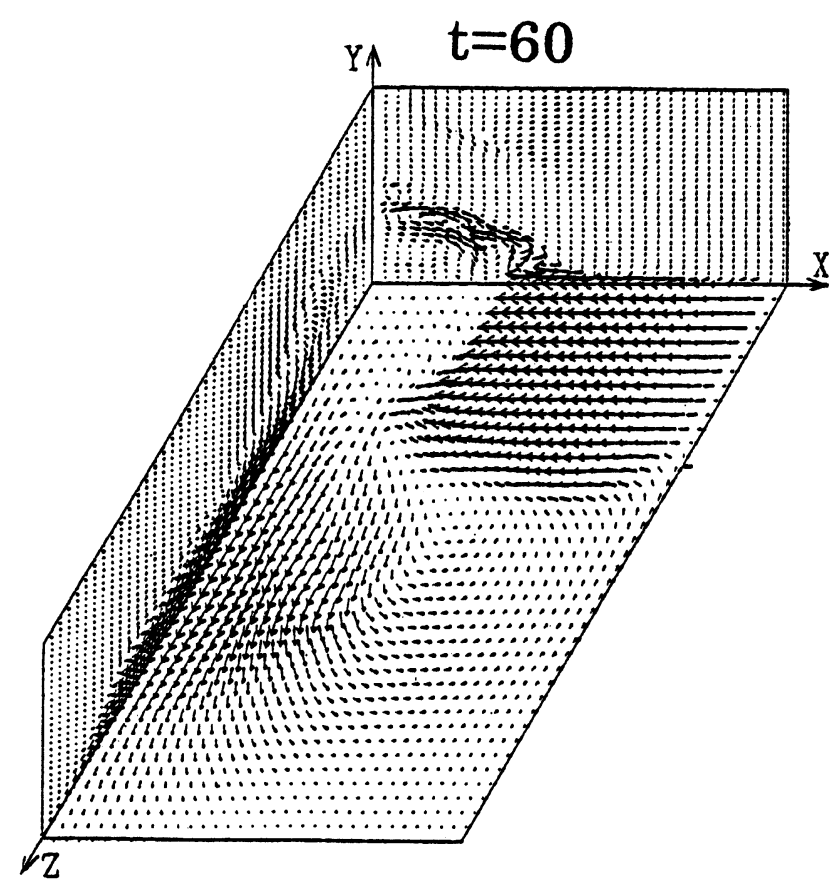

Fig. 7. Plasma flow vectors for the three-dimensional magnetic loop in the $(x, y),(y, z)$, and $(z, x)$ planes.

the interplanetary magnetic field may be reconnected with the dayside magnetosphere, giving rise to the so-called flux transfer event (Lee and $\mathrm{Fu}, 1986$; Scholer, 1989b); also, in the solar corona the emerging magnetic flux may be reconnected with the overlying magnetic field lines (Yokoyama and Shibata, 1995). Hence, the spontaneous fast reconnection model may be applied to an asymmetric current sheet system (Ugai, 2000).

Even in asymmetric situations with a finite sheared field component, we find that the spontaneous fast reconnection mechanism works effectively. As the fast reconnection 


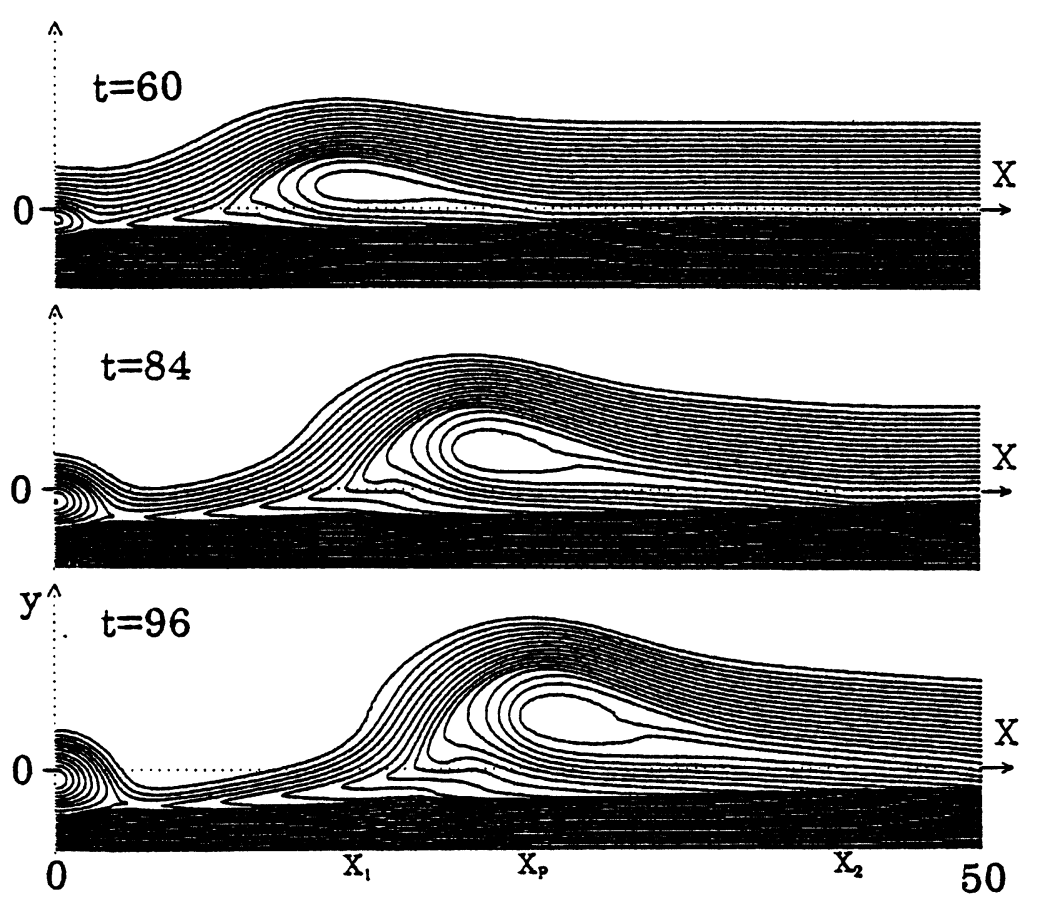

Fig. 8. Magnetic field configurations for the spontaneous fast reconnection evolution in an aymmetric situation.

evolves explosively, a large-scale asymmetric plasmoid swells predominantly in the higher- $\beta$ region of the weaker magnetic field and propagates along the field lines. Figure 8 typically shows the resulting magnetic field configuration, where the central diffusion region (initially located at the origin) is bifurcated into a pair of X neutral points. Even if the $\mathrm{X}$ point moves with time, the enhanced (current-driven) anomalous resistivity always remains localized at the moving $\mathrm{X}$ point, which allows the fast reconnection mechanism to be sustained. We also see that notable erosion of the magnetic field takes place in the region of stronger magnetic field. Apparently, significant magnetic flux transfer takes place in asymmetric situations by the spontaneous fast reconnection evolution.

In accordance with the fast reconnection evolution, the initial current sheet is bifurcated into a pair of asymmetric current layers. The current layer at the lower boundary of stronger magnetic field (Fig. 8) is found to be identified with an ordinary slow shock. On the other hand, the current layer at the upper boundary of weaker magnetic field is of an intermediate-shock like strucure. We find that this structure is transient and propagates in the positive $x$ direction, finally being bifurcated into a finite-amplitude intermediate wave and a slow shock at the upper plasmoid boundary. In this respect, the details can be seen from figures 8 and 10 in Ugai (2000).

\section{Summary and Discussion}

In understanding large dissipative events observed in space plasmas, an essential question is: How can the fast reconnection mechanism involving standing slow shocks evolve and be realized as an eventual solution? In this respect, we have proposed the spontaneous fast reconnection model, which describes a new type of nonlinear instability in a long cur- rent sheet system. High-resolution MHD simulations have demonstrated that the fast reconnection can evolve explosively when the threshold of current-driven anomalous resistivity is sufficiently large; also, the peak reconnection rate attained is sustained during the nonlinear saturation phase. Hence, the spontaneous fast reconnection evolution during the nonlinear saturation phase can be characterized by the localized anomalous resistivity (diffusion region), the attached pair of slow shocks extending outwards, and the large-scale propagating plasmoid.

On the basis of the spontaneous fast reconnection model, distinct plasma processes that are closely related to flare phenomena can readily be demonstrated. First of all, a largescale plasmoid directly results from the spontaneous fast reconnection evolution; in fact, it swells and propagates along the antiparallel field lines in a free space. In the forward half of the plasmoid boundary, the generator effect works, so that the region of compressed magnetic field travels with the plasmoid. Secondly, when the superfast reconnection jet is significantly braked by a magnetic loop, a definite fast shock is formed ahead of the magnetic loop. The fast shock changes its strength oscillatorily, giving rise to notable plasma heating at the loop top as well as at the loop footpoints. Finally, it is demonstrated that the spontaneous fast reconnection model works effectively even in general asymmetric situations, giving rise to drastic magnetic flux transfer.

In summary, we have demonstrated the spontaneous evolution of fast reconnection mechanism as a nonlinear instability. According to this model, a localized (current-driven) anomalous resistivity is essential for the (large-scale) fast reconnection evolution: That is, the associated explosive flare phenomena should be triggered by sudden onset of anomalous resistivity at some localized region in a long current sheet. Apparently, this basic physical concept is quite dif- 
ferent from the one of the conventional externally driven model. In fact, MHD simulations have demonstrated that for a uniform resistivity model the fast reconnection mechanism cannot be set up nor sustained even if the relevant boundary conditions are assumed (Scholer, 1989a; Yokoyama and Shibata, 1994; Ugai, 1994). In actual space systems, the threshold for occurrence of current-driven anomalous resistivities may be very large, so that once the threshold is exceeded locally somewhere in a current sheet, the spontaneous fast reconnection mechanism can explosively build up, leading to collapse of the overall magnetic field system.

Acknowledgments. This work was partially supported by the Grant-in-Aid from the Ministry of Education of Japan, the Radio Atmospheric Science Center of Kyoto University and the SolarTerrestrial Environment Laboratory of Nagoya University. The computer program was run at the Computational Center of Nagoya University.

\section{References}

Kulsrud, R. M., Magnetic reconnection in a magnetohydrodynamic plasma, Phys. Plasmas, 5, 1599-1606, 1998.

Lee, L. C. and Z. F. Fu, A simulation study of magnetic reconnection: Transition from a fast-mode to a slow-mode expansion, J. Geophys. Res., 91, 4551-4556, 1986.

Magara, T. and K. Shibata, Evolution of eruptive flares II. The occurrence of locally enhanced resistivity, Astrophys. J., 514, 456-471, 1999.

Petschek, H. E., Magnetic field annihilation, in AAS-NASA Symposium on the Physics of Solar Flares, NASA Spec. Pub., SP-50, 425-439, 1964.

Priest, E. R. and T. G. Forbes, New models for fast steady state magnetic reconnection, J. Geophys. Res., 91, 5579-5588, 1986.

Sato, T. and T. Hayashi, Externally driven magnetic reconnection and a powerful magnetic energy converter, Phys. Fluids, 22, 1189-1202, 1979.

Scholer, M., Undriven magnetic reconnection in an isolated current sheet, J. Geophys. Res., 94, 8805-8812, 1989a.

Scholer, M., Asymmetric time-dependent and stationary magnetic reconnection at the dayside magnetopause, J. Geophys. Res., 94, 15099-15111, $1989 b$.
Schumacher, J. and B. Kliem, Dynamic current sheets with localized anomalous resistivity, Phys. Plasmas, 3, 4703-4711, 1996.

Shibata, K., MHD simulations of magnetic reconnection and solar flares, in Numerical Astrophysics, edited by S. M. Miyama et al., pp. 303-310, Kluwer Academic Publisher, Netherlands, 1999.

Tsuneta, S., Structure and dynamics of magnetic reconnection in a solar flare, Astrophys. J., 456, 840-849, 1996.

Ugai, M., Self-consistent development of fast magnetic reconnection with anomalous plasma resistivity, Plasma Phys. and Controlled Fusion, 26, 1549-1563, 1984.

Ugai, M., Global dynamics and rapid collapse of an isolated current-sheet system enclosed by free boundaries, Phys. Fluids, 29, 3659-3667, 1986.

Ugai, M., Computer studies on the fast reconnection mechanism under an externally driven boundary condition, Phys. Plasmas, 1, 2853-2863, 1994.

Ugai, M., Computer studies on plasmoid dynamics associated with the spontaneous fast reconnection mechanism, Phys. Plasmas, 2, 3320-3328, 1995.

Ugai, M., Computer studies on dynamics of a large-scale magnetic loop by the spontaneous fast reconnection model, Phys. Plasmas, 3, 4172-4180, 1996.

Ugai, M., Computer studies on the spontaneous fast reconnection model as a nonlinear instability, Phys. Plasmas, 6, 1522-1531, 1999a.

Ugai, M., Basic physical mechanism of reconnection development and magnetic loop dynamics, J. Geophys. Res., 104, 6929-6939, 1999 b.

Ugai, M., Computer simulations of asymmetric spontaneous fast reconnection, Phys. Plasmas, 7, 867-874, 2000.

Ugai, M. and T. Tsuda, Magnetic field-line reconnexion by localized enhancement of resistivity. Part 1. Evolution in a compressible MHD fluid, J. Plasma Phys., 17, 337-356, 1977.

Ugai, M. and W. B. Wang, Computer simulations on three-dimensional plasmoid dynamics by the spontaneous fast reconnection model, J. Geophys Res., 103, 4573-4586, 1998.

Vasyliunas, V. M., Theoretical models of magnetic field line merging, 1, Rev. Geophys., 13, 303-336, 1975.

Yokoyama, T. and K. Shibata, What is the condition for fast magentic reconnection, Astrophys. J., 436, L197-L200, 1994.

Yokoyama, T. and K. Shibata, Magnetic reconnection as the origin of X-ray jets and $\mathrm{H} \alpha$ surges on the Sun, Nature, 375, 42-44, 1995.

M. Ugai (e-mail: ugai@cs.ehime-u.ac.jp) 